\title{
DESEMPENHO TECNOLÓGICO DE PAPÉIS PRODUZIDOS COM ADIÇÃO DE NANOFIBRILAS DE CELULOSE DE Eucalyptus
}

\author{
TECHNOLOGICAL PERFORMANCE OF PAPER PRODUCED WITH Eucalyptus CELLULOSE \\ NANOFIBRILS ADDITION \\ Filipe Santos da Silva ${ }^{1}$, Henrique Römer Schulz ${ }^{2}$, Andrey Pereira Acosta ${ }^{3}$, \\ Gabriel Valim Cardoso ${ }^{4}$ \\ 1, 2, 3, 4 Universidade Federal De Pelotas, Pelotas, Rio Grande do Sul, Brasil -filipissantos.s@gmail.com, \\ henriqueschulz09@hotmail.com, andreysvp@gmail.com \& gabriel.valim.cardoso@gmail.com
}

\section{RESUMO}

\begin{abstract}
A produção de papel possuí grande destaque no setor industrial madeireiro, porém existe a necessidade de tratamentos que visem a melhora nos seus parâmetros tecnológicos. Nesse contexto pode ser citado, a inserção de celulose nanofibrilada na produção de papéis, pois devido as significativas melhoras nas propriedades mecânicas e físicas tem por sua natureza ser biodegradável. Diante disto, este trabalho foi desenvolvido com o objetivo de avaliar o efeito da adição de diferentes concentrações de nanofibrilas de celulose (NFC) nas propriedades mecânicas de folhas de papéisPara isso, foram confeccionadas 14 folhas com celulose de fibra de Eucalyptus, em duplicata. Para visualizar a estrutura e as dimensões das fibras e nanofibrilas de celulose foram usadas as técnicas de microscopia eletrônica de varredura em um dos tratamentos. Avaliaram-se as propriedades físicas e mecânicas dos papeis confeccionados com incorporação de nanofibrilas de celulose nas porcentagens de 0,2,4,8,16,32 e 64\%. A adição de nanofibrilas de celulose no papel proporcionou a redução na espessura e aumento da densidade aparente. Os índices de resistência à tração, ao estouro e elongamento aumentaram significativamente com a adição de nanocelulose ao papel, devido às interações intra e intermoleculares.
\end{abstract}

PALAVRAS-CHAVE: MEV, Nanocelulose, Produção de papel, Testes físicos, Testes mecânicos.

\section{ABSTRACT}

The paper production has great prominence in the timber industry, but there is a need for treatments aimed at improving its technological parameters. In this context it can be mentioned, the insertion of nanofibrillated cellulose in paper production, because due to the significant improvements in mechanical and physical properties has by its nature be biodegradable. Therefore, this work was developed with the objective of evaluating the effect of the addition of different concentrations of cellulose nanofibrils (NFC) on the mechanical properties of paper sheets. Thus, 14 sheets were made with duplicate Eucalyptus fiber cellulose. To visualize the structure and dimensions of cellulose fibers and nanofibrils, scanning electron microscopy techniques were used in one of the treatments. The physical and mechanical properties of papers made with incorporation of cellulose nanofibrils in the percentages of $0,2,4,8,16,32$ and $64 \%$ were evaluated. The addition of cellulose nanofibrils to the paper provided a reduction in thickness and increased bulk density. Tensile strength, bursting and elongation rates increased significantly with the addition of nanocellulose to the paper due to intra and intermolecular interactions.

KEYWORDS: MEV, Nanocellulose, Paper production, Physical tests, Mechanical tests. 


\section{INTRODUÇÃO}

Tendo em vista o crescimento notório do setor de celulose e papel, e o aumento de consumo mundial de papel, observa-se a necessidade cada vez maior do desenvolvimento de novas tecnologias que venham a contribuir para o melhoramento da qualidade dos produtos e o aumento da produção do setor dependente dessa fonte (MONTEBELLO \& BACHA, 2013).

Com os avanços no manejo florestal, a indústria brasileira de celulose e papel tornou-se mundialmente competitiva, com o Brasil entre os principais produtores. De acordo com CARVALHO (2017), e as inovações tecnológicas feitas a partir de derivados de fontes renováveis têm recebido atenção dos cientistas nos últimos anos. Nesse contexto, pode ser citado a utilização das nanofibrilas de celulose.

A nanofibrila é uma matéria prima natural e renovável (HENTZE, 2010), uniforme e altamente cristalina (YANO et al., 2007) obtida pelo processo de fibrilação mecânica da celulose, que gera uma desintegração da parede celular da madeira (HENRIKSSON, 2008), e modifica as suas propriedades estruturais e de superfície (YANO; NAKAGAITO, 2004), bem como a sua dimensão.

As nanofibrilas de celulose (NFCs) estão se tornando um tema de grande interesse para pesquisadores de muitos campos científicos e tecnológicos. A quantidade de publicações que envolvem a produção, caracterização e aplicação de NFCs tem aumentado ao longo dos últimos anos. Estes estudos são principalmente com NFCs fabricadas a partir de fibras de madeira branqueadas.

A celulose nanofibrilada a partir da biomassa recentemente ganhou atenção devido à sua natureza biodegradável, altas propriedades mecânicas, valor econômico e renovabilidade.

Oriundas de processos mecânicos de processamento da própria celulose, as nanofibrilas de celulose são incorporadas ao papel com o objetivo de aumentar as propriedades e dar um melhor acabamento. Devido as suas dimensões em escala nanométricas e alta área superficial, possuem um potencial de ocupar pequenos espaços vazios e proporcionar um melhor entrelaçamento das fibras, tornando-se um aditivo para o papel (POTULSKI, 2014).

Os estudos feitos sobre as propriedades da celulose nanofibrilada mostram que, comparativamente com fibras refinadas, está possui uma capacidade muito maior de estabelecer ligações por pontes de hidrogênio. Ainda assim, este material continua a ser celulose, ou seja, um produto natural biodegradável. Desta forma, a sua aplicação como material de reforço torna-se um interesse em estudos de várias áreas (FIGUEIREDO, 2006).

O presente trabalho teve como objetivo, verificar as mudanças geradas nas folhas de papéis produzidas com celulose de Eucalyptus, relacionadas a incorporação de nanofribrilas em diferentes porcentagens $(0,2,4,8,16,32$ e 64\%). Sendo avaliado os parâmetros tecnológicos por meio da microscopia eletrônica de varredura (MEV), análises das propriedades mecânicas, resistência à tração, elongamento e resistência ao estouro, e as propriedades físicas, resistência a passagem do ar (Gurley), espessura e gramatura, foram determinadas seguindo as Normas ISSO.

\section{MATERIAL E MÉTODOS}

\section{Produção dos papéis}

O trabalho foi desenvolvimento no Laboratório de Celulose e Papel do Curso de Engenharia Industrial Madeireira da Universidade Federal de Pelotas. Foi utilizada polpa celulósica branqueada na forma de cartão, da madeira de Eucalyptus ssp, cedidas pela empresa Celulose Riograndese CMPC - Guaíba, RS, da qual foram obtidas nanofibrilas de celulose.

Para a produção das nanofibrilas de celulose, foi utilizado um refinador de discos tipo Bauer modelo MD3000, utilizando fibras de celulose branqueada de eucalipto. $O$ processo mecânico de nanofibrilação consiste em refinar as fibras por um período de 3 horas com consistência de $2 \%$. Após a produção as nanofibrilas foram mantidas sob refrigeração na consistência de 2,2\% para posterior aplicação nos papéis.

Foi utilizada uma amostra para caracterização por meio de microscopia eletrônica de varredura (MEV) no Centro de Microscopia Eletrônica da Universidade Federal de Rio Grande (CEME-SUL/FURG), para que fosse feita a confirmação da dimensão nanométricas das fibrilas.

Pesaram-se 2 gramas úmidas de amostra de papel em uma placa de petri, realizado em duplicata e acondicionado em estufa por 24 horas, sendo retirado da estufa e pesado novamente, descontando o peso da placa foi possível obter o peso da amostra seca, dessa forma aplicando na seguinte Equação (1), equação do teor de umidade.

$$
\mathrm{TU}=\left(\frac{\mathrm{PU}-\mathrm{PS}}{\mathrm{PU}}\right) * 100
$$

em que: $\mathrm{TU}=$ teor de umidade (\%); PU = amostra úmida (g); PS = amostra seca (g). 
Em seguida, pesaram-se 24 gramas secas de celulose branqueada. As amostras ficaram em saturando em 2 litros de água destilada durante 48 horas, para se obter uma melhor individualização destas.

Então, com a celulose hidratada, foi realizado o processo de desagregação das fibras utilizando um desagregador automático de acordo com as normas técnicas ABNT NBR ISO 5263-1 e 5263-2, permanecendo por 10.000 rotações para desagregação completa das fibras.

A polpa obtida no processo de desagregação foi diluída em um galão com 8 litros de água destilada para alcançar 0,3\% de consistência, calculado pela Equação 2.

$$
\mathrm{Cs}=\left(\frac{\mathrm{M}}{\mathrm{V}}\right) * 100
$$

em que: $C s=$ consistência do material (\%); $M=$ massa (g); V = quantidade de água para a diluição do material (I).

Para a formação dos papéis foi usado o equipamento formador de folhas de laboratório, modelo FF/C. O formador de uma tela com área de 0,0198 $\mathrm{m}^{2}$, sendo necessária uma massa de 1,2 gramas de fibras para se obter a gramatura desejada $(60 \mathrm{~g} / \mathrm{m} 2)$. A quantidade de nanofibrilas a ser adicionada em cada concentração foi obtida calculando-se a percentagem destas em relação a massa de 1,2 gramas.

Foi definido a formação de folhas com gramatura de 60 $\mathrm{g} / \mathrm{m}^{2}$, nas quais foram adicionadas concentrações de nanofibrilas de $0,2,4,8,16,32$ e 64\% conforme as tabelas 1 e 2. Após a formação das folhas, o material foi levado para prensa pneumática para folhas de laboratório Regmed, modelo SP-21, de acordo com ABNT NBR ISO $5269 / 1$. Por final, levado à câmara climatizada para a climatização das folhas de papel, adquirindo teor de umidade e peso constante, segundo a norma ISO 187:1990.

\section{Caracterização das propriedades dos papéis}

Os papéis produzidos sem adição e com $16 \%$ de nanofibrilas foram caracterizados por MEV. Para isso, foram preparadas as superfícies secas dos corpos de prova com recobrimento por pó de ouro, o qual recobre a superfície do papel, funcionando como um condutor para a passagem de feixes de elétrons, assim podem ser obtidas imagens com aumento de 100 e 15000 vezes por meio de um microscópio eletrônico de varredura da marca Jeol, modelo JSM 6610, localizado no Centro de Microscopia Eletrônica da Universidade Federal de Rio Grande (CEMESUL/FURG).
O grau de refinação mede a resistência à drenagem da polpa celulósica e foi determinado seguindo a norma ISO $5267 / 1$, através do equipamento Schopper Riegler.

Foram determinadas as propriedades das folhas de papel na empresa CMPC Celulose Riograndense, localizada em Guaíba no estado do Rio Grande do Sul, onde foram feitas as análises das propriedades mecânicas, resistência à tração, elongamento e resistência ao estouro, e as propriedades físicas, resistência a passagem do ar (Gurley), espessura e gramatura, foram determinadas seguindo as Normas ISO descritas na Tabela 1.

Tabela 1. Propriedades mecânicas e físicas analisadas com base nas respectivas normas.

\begin{tabular}{cccc}
\hline $\begin{array}{c}\text { Propriedades } \\
\text { mecânicas }\end{array}$ & Normas & $\begin{array}{c}\text { Propriedades } \\
\text { físicas }\end{array}$ & Normas \\
\hline Resistência & ISO 2758: & Gramatura & ISO 536: \\
ao Estouro & 2001 & & 1995 \\
Resistência & ISO 1924/2: & Espessura & ISO 534: \\
à Tração & 2006 & Resistência & ISO 5636- \\
Elongamento & ISO 1924/3: & ao ar & $5: 2013$ \\
\hline
\end{tabular}

Para verificar a influência da adição de nanofibrilas de celulose nas propriedades de folhas de papel, os valores obtidos foram sujeitos à análise estatística, utilizando o software STATGRAPHICS Centurion XVII. As propriedades físicas e mecânicas dos papeis produzidos, foram submetidas à análise de variância - ANOVA, aplicou-se o teste Tukey ao nível de $5 \%$ de significância para comparação entre as médias.

\section{RESULTADOS E DISCUSSÃO}

A Figura 1 mostra o comparativo do papel sem adição de nanofibrilas (A) e com adição de $16 \%$ de NFC (B), visualizados mediante a utilização da técnica de microscopia eletrônica de varredura (MEV).

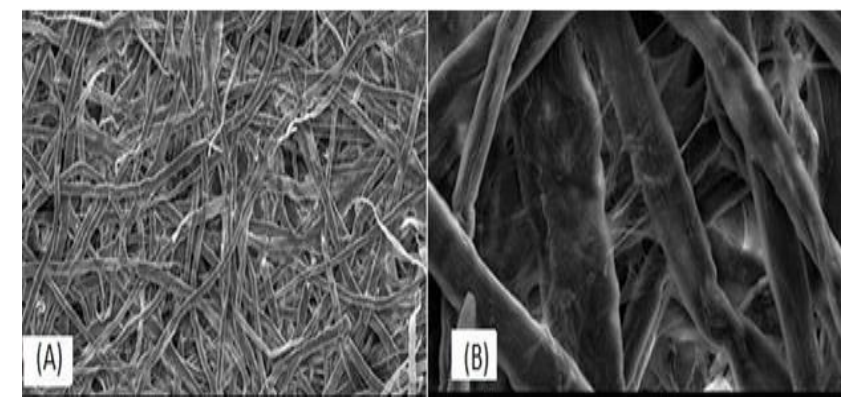

Figura 1. (A) Papel sem nanofibrilas a 0\%; (B). Papel com nanofibrilas a $16 \%$. 
A presença de nanofibrilas confirma a capacidade de entrelaçamento e preenchimento dos espaços vazios. Essa vantagem se dá devido às características da celulose nanofibrilada de formar ligações entre as fibras, promovendo assim o aumento na resistência das folhas de papel. A celulose nanofibrilada, com diâmetros menores que 100 nanometros, foi exposta na Figura 2.

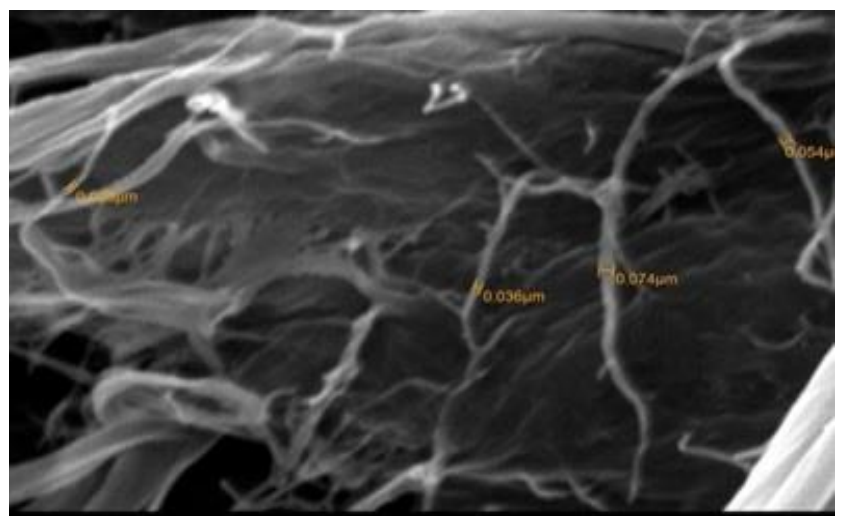

Figura 2. Nanofibras de celulose.

Os valores de grau Schopper Riegler ( ${ }^{\circ} \mathrm{SR}$ ) das polpas celulósicas de fibras virgens de Eucalyptus sp. sem e com adição de 2, 4, 8, 16, 32 e $64 \%$ de celulose nanofibrilada. 0 Grau Schopper Riegler foi influenciado significativamente conforme o aumento da adição de NFCs à polpa celulósica. O ${ }^{\circ} \mathrm{SR}$ está inversamente relacionado a drenabilidade da polpa celulósica, no qual o aumento no Grau Schopper indicou redução da capacidade de drenagem da polpa.

Os maiores aumentos em resistência à drenagem podem ser devido ao fato da celulose nanofibrilada ter promovido a maior quantidade de ligações de hidrogênio com as moléculas de água, por causa de sua superfície da parede celular mais exposta, e assim contribuiu para uma maior hidratação, consequentemente maior flexibilidade e melhor arranjo das fibras na formação da folha, o que poderia refletir consideravelmente nas propriedades físico- mecânicas do papel.

A redução do oSR de 19 para 14 quando aplicado $2 \%$ de NFC em relação ao tratamento sem NFC, se deve à reduzida quantidade de nanofibrilas adicionadas neste tratamento as quais acabam passando pela tela de desaguamento do equipamento, causando perda de massa o que gera aumento de velocidade de água escoada, que resultou no menor valor de oSR. O SR obtidos com a adição de 16, 32 e $64 \%$ equivalem a resultados de polpas com elevado refino, indicando que as NFC podem substituir este processo.

Pode-se observar que a resistência à drenagem nos diz que a adição de celulose nanofibrilada possa auxiliar ou até reduzir parcialmente o processo de refinação, que nos processos convencionais tem como função o aumento da resistência à drenagem bem como o aumento das propriedades de resistência do papel (RAZZOLINI, 1994; KALIA et al., 2014).

Os valores médios obtidos para as propriedades físicas de gramatura, espessura, e permeância ao ar, e dos testes mecânicos de Resistência a Tração, Resistência ao Estouro e Elongamento obtidos pelo ANOVA e analisados com o teste de médias Tukey, estão presentes nas Tabelas 2 e 3, respectivamente.

Tabela 2. Propriedades físicas dos papeis sem e com adição de nanofibrilas.

\begin{tabular}{cccc}
\hline $\begin{array}{c}\text { Tratamento } \\
(\%)\end{array}$ & $\begin{array}{c}\text { Gramatura } \\
\left(\mathbf{g} / \mathbf{m}^{\mathbf{2}}\right)\end{array}$ & $\begin{array}{c}\text { Espessura } \\
(\boldsymbol{\mu m})\end{array}$ & $\begin{array}{c}\text { Gurley } \\
\left(\mathbf{s} / \mathbf{c m}^{\mathbf{3}}\right)\end{array}$ \\
\hline 0 & $60,37 \mathrm{a}$ & $81,79 \mathrm{c}$ & $3,3 \mathrm{a}$ \\
2 & $60,14 \mathrm{a}$ & $82,27 \mathrm{c}$ & $3,7 \mathrm{a}$ \\
4 & $60,64 \mathrm{a}$ & $81,38 \mathrm{c}$ & $4,8 \mathrm{a}$ \\
8 & $61,10 \mathrm{a}$ & $79,37 \mathrm{bc}$ & $7,8 \mathrm{~b}$ \\
16 & $60,46 \mathrm{a}$ & $75,77 \mathrm{~b}$ & $23,00 \mathrm{c}$ \\
32 & $59,00 \mathrm{a}$ & $68,47 \mathrm{a}$ & $137,8 \mathrm{~d}$ \\
64 & $57,73 \mathrm{a}$ & $62,84 \mathrm{a}$ & $1094,3 \mathrm{e}$ \\
\hline
\end{tabular}

Médias seguidas pela mesma letra, na mesma coluna, não diferem estatisticamente entre si ao nível de 5\% de significância pelo teste Tukey.

A propriedade de tração é influenciada pelo comprimento das fibras e, principalmente, ligação entre as fibras e pela formação e estrutura da folha (FERREIRA, 2008), características que justificam a tendência de aumento de resistência da folha de papel com a incorporação de NFCs. Em que apresentou uma variação de 14,597 a $59,996 \mathrm{KNm} / \mathrm{Kg}$. Isso ocorre devido às características das NFCs que tem grande capacidade de ligação entre as fibras, promovendo assim uma melhor formação e folhas mais homogêneas.

Tabela 3. Propriedades mecânicas dos papeis sem e com adição de nanofibrilas.

\begin{tabular}{cccc}
\hline $\begin{array}{c}\text { Tratamento } \\
\text { (\%) }\end{array}$ & $\begin{array}{c}\text { Tração } \\
\text { (KNm/Kg) }\end{array}$ & $\begin{array}{c}\text { Estouro } \\
\text { (Kpa.m } \mathbf{~} / \mathbf{g})\end{array}$ & $\begin{array}{c}\text { Elongamento } \\
\text { (\%) }\end{array}$ \\
\hline 0 & $14,597 \mathrm{a}$ & $0,972 \mathrm{a}$ & $1,432 \mathrm{a}$ \\
\hline 2 & $16,584 \mathrm{a}$ & $1,012 \mathrm{a}$ & $1,758 \mathrm{a}$ \\
\hline 4 & $17,491 \mathrm{a}$ & $1,014 \mathrm{a}$ & $2,050 \mathrm{a}$ \\
\hline 8 & $23,433 \mathrm{~b}$ & $1,266 \mathrm{~b}$ & $2,906 \mathrm{~b}$ \\
\hline 16 & $30,123 \mathrm{c}$ & $1,873 \mathrm{c}$ & $3,829 \mathrm{c}$ \\
\hline 32 & $42,399 \mathrm{~d}$ & $3,232 \mathrm{~d}$ & $4,800 \mathrm{~d}$ \\
\hline 64 & $59,996 \mathrm{e}$ & $5,653 \mathrm{e}$ & $6,845 \mathrm{e}$ \\
\hline
\end{tabular}

Médias seguidas pela mesma letra, na mesma coluna, não diferem estatisticamente entre si ao nível de 5\% de significância pelo teste Tukey. 
A resistência ao estouro apresentou uma variação de 0,972 a 5,653 $\mathrm{KPam}^{2} / \mathrm{g}$, isso significa uma tendência de crescimento da resistência com a adição das NFCs, mesma tendência observada, visivelmente, na resistência a tração.

Provavelmente, a tendência observada para a resistência ao estouro tenha sido semelhante à observada para a resistência a tração, sendo que as duas propriedades foram afetadas pelos mesmos fatores, principalmente, a capacidade de ligação entre as fibras, o principal entre eles. Schulz et al. (2019) em seu estudo, observou o efeito significativo do refino na melhoria de parâmetros físicos e mecânicos em papéis produzido com fibras virgens e recicladas de Eucalyptus.

A resistência ao elongamento é a medida da distensão do papel quando estendido até a ruptura em percentuais. Por sua vez, tem parecença com o teste de tração, onde também teve influência significativa quanto ao aumento de dosagens em porcentagens de nanofibrilas na polpa celulósica e variou de 1,432 a 6,845 \%. Ocorre que acrescentando nanofibrilas como aditivo principal em sua composição, origina um material de maior capacidade em resistir quando forças são aplicadas a fim de se obter sua ruptura. Potulski et al. (2014), corrobora com o relatado nesse estudo, verificando a melhora significativa nos parâmetros mecânicos com a inserção de microfibrilas na produção de papéis.

Analisando os valores médios obtidos através do teste Gurley o tratamento com $16 \%$ de nanofibrilas apresentou o valor de $23\left(\mathrm{~s} / \mathrm{cm}^{3}\right)$, sendo um valor intermediário entre todos os tratamentos analisados. Quanto maior o valor de tal parâmetro, maior a dificuldade de fabricação do papel com adição das nanofibrilas.

Com isso, observou-se que a adição das NFCs influencia positivamente nas propriedades de resistência mecânicas dos papeis analisados, porém é importante que esse tipo de tratamento seja realizado considerando alguns parâmetros de grande relevância, como a quantidade a ser adicionada, para que não atue inversamente ao objetivo proposto, de incrementar os índices de resistência e ser viável na utilização em indústrias do ramo madeireiro.

\section{CONCLUSÕES}

Com isso conclui-se em base nas imagens microscópicas mostradas detalhadamente que a concentração de $16 \%$ de nanofibrilas afetaram na microestrutura das folhas de papéis. Quanto às propriedades físicas do papel, gramatura, espessura e resistência ao ar, estas foram influenciadas tanto pelo processo de fabricação quanto pela adição das nanofibrilas de celulose.

As propriedades mecânicas, resistência à tração, ao estouro e ao elongamento, foram influenciadas pela adição das nanofibrilas de celulose. Entre as propriedades mecânicas, a resistência à tração foi que sofreu maior influência da adição das nanofibrilas de celulose.

$A$ adição de nanofibrilas de celulose elevou os resultados de Grau Schopper Riegler sem a necessidade de refino das fibras. A adição de $16 \%$ de nanofibrilas apresentou o melhor potencial de aplicação industrial, considerando o baixo valor de permanência ao ar (Gurley). Relacionado com a drenagem de água na formação da folha, aliado aos elevados ganhos de propriedades mecânicas.

As nanofibrilas de celulose podem se tornar uma alternativa viável na substituição de outros materiais, ou até mesmo para fim de melhorar a qualidade dos produtos utilizados atualmente.

\section{REFERÊNCIAS}

CARVALHO, A.J.F. Nanofibras de celulose, plástico e acrílico: enfim juntos. Revista Madeira Total, v.35, n.8, p.1-28, 2017.

FERREIRA, P. Propriedades do papel: Ciência e Tecnologia da pasta do papel. Universidade de Coimbra, 2008.

FIGUEIREDO, J.L. As inimagináveis potencialidades da nanotecnologia. Mundus, 2006. Disponível em: http://w3.ualg.pt/ jlongras/Entrevista-Mundus.pdf.

HENRIKSSON, M. Cellulose nanofibril networks and composites. 2008. 51p. (Tese de doutorado).

HENTZE, H.P. From nanocellulose science towards applications. Helsinki: VTT - Technical Research Center of Finland, 2010. Disponível em: https://www.vtt.fi/Documents/NFCApplications _HPH.pdf.

ISO $1924-2$ e 3. Paper and board - Determination of tensile properties. 2008.

ISO 2758. Paper - Determination of bursting strength. 2014.

ISO 5263-1. Pulps - Laboratory wet disintegration -- Part 1: Disintegration of chemical pulps. 2004.

ISO 5263-2. Pulps - Laboratory wet disintegration, 2004.

ISO 187: Paper, board and pulps - Standard atmosphere for conditioning and testing and procedure for monitoring the atmosphere and conditioning of samples. 1990.

ISO 534. Paper and board - Determination of thickness, density and specific volume. 2005.

ISO 536. Paper and board - Determination of grammage. 1995. 
ISO 5636-5. Paper and board - Determination of air permeance. 2013.

ISO 5267-1. Pulps - Determination of drainability - Part 1: Schopper-Riegler method. 1999.

ISO 5269-1. Pulps - Preparation of laboratory sheets for physical testing. 2006.

KALIA, S. et al. Nanofibrillated cellulose: surface modification and potential applications. Colloid and Polymer Science, v.292, p.531, 2014.

MONTEBELLO, A.E.S., BACHA, C.J.C. Impactos da reestruturação do setor de celulose e papel no Brasil sobre o desempenho de suas indústrias. Estudos Econômicos, v.43, n.1, p.109-137, 2013.

SCHULZ, H.R. et al. Caracterização de propriedades tecnológicas de papéis de fibra virgem e reciclada após refinamento. BIOFIX Scientific Journal, v.4, n.2, p.160-165, 2019.

POTULSKI, D.C. et al. Influência da incorporação de celulose microfibrilada nas propriedades de resistência mecânicas do papel. Scientia Forestalis, v.42, n.103, p.345-351, 2014.

RAZZOLINI, F.C. Técnicas de fabricação de papéis e cartões para embalagem. ABTCP, 1994.

YANO, H.; NAKAGAITO, A.N. The effect of morphological changes from pulp fiber towards nano-scale fibrillated cellulose on the mechanical properties of high- strength plant fiber based composites. Applied Physics A, v.78, n.4, p.547-552, 2004.

Recebido em 27-01-2020 Aceito em 30-09-2019 